\title{
SYNTHESIS AND MICROBIOLOGICAL EVALUATION OF 1-(4-METHYL-6-NITRO-2H-BENZO[B] [1,4]THIAZINE-3(4H)-YLIDENE)HYDRAZINE-1,1-DIOXIDE DERIVATIVES
}

\author{
MONIKA MAHESHWARI*, ANJU GOYAL \\ Department of Pharmaceutical Chemistry, B N Institute of Pharmaceutical Sciences, Sevashram, Udaipur, Rajasthan, India. \\ Email: mona30mph@gmail.com
}

Received: 11 April 2017, Revised and Accepted: 09 June 2017

\section{ABSTRACT}

Objective: The objective of this work was to synthesize and evaluate antimicrobial properties of 1-(4-methyl-6-nitro-2 $H$-benzo[b][1,4]thiazine3(4H)-ylidene)hydrazine-1,1-dioxide derivatives.

Methods: These new compounds were synthesized by methylation in 4-N and reacted with hydrazine derivatives and oxidized at the sulfur atom by $30 \%$ hydrogen peroxide to obtain sulfones. All the synthesized compounds were evaluated for antimicrobial activity using the disc diffusion method.

Results: The Fourier transform infrared, ${ }^{1} \mathrm{H}$ nuclear magnetic resonance (NMR), ${ }^{13} \mathrm{CNMR}$, and mass studies confirm the synthesis of some new 1 - $(4-\mathrm{methyl}$ 6-nitro-2H-benzo $[b][1,4]$ thiazine-3(4H)-ylidene)hydrazine-1,1-dioxide derivatives. Compound $6 \mathrm{f}$ showed the potent antimicrobial activity.

Conclusion: Result obtained in this research work clearly indicated that the compound $6 \mathrm{f}$ having methyl at 2 position and nitro groups at $2^{\prime}$ and $4^{\prime}$ position showed the most potent antimicrobial activity.

Keywords: 1,4-Benzothiazines, Sulfones, Hydrazines, Antimicrobial activity.

(C) 2017 The Authors. Published by Innovare Academic Sciences Pvt Ltd. This is an open access article under the CC BY license (http://creativecommons. org/licenses/by/4. 0/) DOI: http://dx.doi.org/10.22159/ajpcr.2017.v10i9.19093

\section{INTRODUCTION}

Research in the synthetic chemistry of 1,4-benzothiazine derivatives during the past few decades was mainly attributed to their unique chemical, physical, and biological properties [1-7]. The synthesis of the sulfoxide system, many sulfones have been shown to exhibit biological activity for the industrial and pharmacological applications $[8,9]$. The oxidation of sulfide linkage in 1,4-benzothiazines to dioxide leads to a significant class of heterocyclic sulfones from medicinal and structural aspects. Alteration of benzothiazine into sulfone has provided an opportunity to study the changes in infrared and nuclear magnetic resonance (NMR) spectra caused by the conversion of the sulfide linkage to sulfones.

In the worldwide as well as in the developing countries, the most human death occurs due to infectious bacterial disease. Drug resistance in human pathogenic microbes has developed due to the indiscriminate use of the commercial antimicrobial drugs for the treatment of the infectious disease. Drug resistance is the major obstacle of this era which is leading toward mortality and morbidity. This condition has been enforced to the researcher to investigate for the new antimicrobial substance which is more efficient and having lesser side effect with improved physical properties. The alkylation of 4-N position of $2 \mathrm{H}$-benzo[1,4] thiazine's affords bactericidal and antifungal derivatives. With the aim to investigate more potent antimicrobial activity of structurally related compounds, several 1-(4-methyl-6nitro-2 $H$-benzo[b][1,4] thiazin-3(4H)-ylidene)hydrazine-1,1-dioxide derivatives $6 a-f$ were synthesized (Fig. 1). The synthesis of compounds 6a-f started from the treatment of 2-chloro-5-nitroaniline 1 with sodium sulfide and sulfur gave sodium-2-amino-4-nitrobenzenethiol 2, which was cyclised with $\beta$-heloesters in ethanolic solution [10] to yield $2 H$-benzo[b][1,4]thiazin-3(4H)-one derivatives 3a-b. Compounds $4 \mathrm{a}$-b were synthesized by methylation of compounds $3 a-b$ by methyl iodide in dimethyl sulfoxide (DMSO)/ethanol [11], which was refluxed with some nitrogen containing nucleophilic hydrazines in methanol [12] to yield 1-(4-methyl-6-nitro-2H-benzo[b][1,4]thiazine-3-yl)hydrazines 5a-f. The further step, that is, the oxidation of the sulfur, was usually performed with $30 \%$ hydrogen peroxide in glacial acetic acid [13] to produce their sulfones $6 \mathrm{a}-\mathrm{f}$. The newly synthesized compounds have been screened for antibacterial and antifungal activity by disc diffusion method [14].

\section{METHODS}

Chemistry

All the chemicals used were of analytical grade. Melting points were determined in open capillary tubes and are uncorrected. Purity of the compounds was checked by thin layer chromatography on silica gel plates with a $0.2 \mathrm{~mm}$ thickness. Compounds were powdered, mixed with $\mathrm{KBr}$ at $1 \%$ concentration, and pressed into pellets before IR spectra be recorded on Bruker FT/IR Vertex spectrometer. ${ }^{1} \mathrm{H}$ - and ${ }^{13} \mathrm{C}$-NMR spectra were recorded on a Bruker Avance II $400 \mathrm{NMR}$ spectrometer using DMSO- $\mathrm{d}_{6}$ as solvent, TMS as an internal standard and the chemical shifts $(\delta)$ are expressed in parts per million (ppm), and coupling constants (J) are given in hertz (Hz). Mass spectra were recorded on a Waters, Q-TOF MS-ES spectrometer. Elemental analysis was done on Carlo Erba 1108 elemental analyzer.

The synthesis, physical, and analytical properties of compounds 2, 3a, and $4 \mathrm{a}$ has been previously described $[10,11]$.

Synthesis of 2-methyl-6-nitro-2-H-benzo[b][1,4] thiazin-3-one (3b) Sodium-2-amino-4-nitrobenzenethiol (1.2 g, $0.01 \mathrm{~mol}$ ) (2) and methyl2-chloropropionate $(1.1 \mathrm{~g}, 0.01 \mathrm{~mol})$ were dissolved in $30 \mathrm{ml}$ ethanol. About $5 \mathrm{ml}$ of $10 \% \mathrm{NaOH}$ was added and refluxed for 3 hrs. Product was poured in ice, washed with water, and recrystallized from ethanol to obtain compound 3 b. Light yellow crystal, yield, $93 \%$; m.p. $174-175^{\circ} \mathrm{C}$; $\mathrm{R}_{\mathrm{f}} 0.89$ (toluene-ethyl acetate, $\left.7: 5\right)$; ultraviolet (UV) (DMSO) $\lambda_{\text {max }}(\log \varepsilon$ ) 346 (4.66) nm; IR (u cm ${ }^{-1}$ ): 3360, 2924, 1671, 1578, 1392, 650; ${ }^{1} \mathrm{H}-\mathrm{NMR}$ (d ppm, DMSO-d ) 1.5 (d, 3H, J=7 Hz, CH-CH $), 3.7$ (q, 1H, H-2), 7.6 (d, $1 \mathrm{H}, \mathrm{J}=8.4 \mathrm{~Hz}, \mathrm{H}-8), 7.9(\mathrm{dd}, 1 \mathrm{H}, \mathrm{J}=8.6$ and $2.2 \mathrm{~Hz}, \mathrm{H}-7), 8.1(\mathrm{~d}, 1 \mathrm{H}$, $\mathrm{J}=2.4 \mathrm{~Hz}, \mathrm{H}-5), 10.95$ (s, $1 \mathrm{H}, \mathrm{NH}) ;{ }^{13} \mathrm{C}-\mathrm{NMR}(\mathrm{d}$ ppm, DMSO-d 6$): 19.9\left(\mathrm{CH}_{3}\right.$, 
$\mathrm{CH}^{-\mathrm{CH}_{3}}$ ), 50.5 (CH, C-2), 115.7 (CH, C-5), 116.9 (CH, C-7), 127.9 (CH, C-8), 131.4 (C, C-9), 143.3 (C, C-10), 145.0 (C, C-6), 169.4 (C, C-3); ESMS m/z (\%): 224 (55), 195 (38), 181 (100), 143 (24), 95 (12). Analysis calculated for $\mathrm{C}_{9} \mathrm{H}_{8} \mathrm{~N}_{2} \mathrm{O}_{3} \mathrm{~S}: \mathrm{C}, 48.21 ; \mathrm{H}, 3.60 ; \mathrm{N}, 12.49 ; \mathrm{S}, 14.30$. Found: C, 48.23; H, 3.58; N, 12.53; S, 14.31 .

\section{Synthesis of 2,4-dimethyl-6-nitro-2-H-benzo[b][1,4]thiazin-3-one (4b)}

2-Methyl-6-nitro-2- $H$-benzo[b][1,4]thiazin-3(4H)-one 3b $(2.4 \mathrm{~g}, 0.01$ $\mathrm{mol})$ and potassium hydroxide $(1.1 \mathrm{~g}, 0.02 \mathrm{~mol})$ were dissolved in DMSO $(20 \mathrm{ml})$ and ethanol $(25 \mathrm{ml})$. The mixture was stirred for 10 minutes before methyl iodide $(1.2 \mathrm{ml}, 0.02 \mathrm{~mol})$ was added. Solution was heated at $50^{\circ} \mathrm{C}$ with stirring for $15 \mathrm{hrs}$. After cooling, water was added and the organic phase was extracted with cyclohexane $(3 \times 50 \mathrm{ml})$ and purified by column chromatography on silica gel with a mixture of tolueneethyl acetate (8:2) as eluent. Yellow oil, yield, $80 \% ; R_{f}, 0.52$ (tolueneethyl acetate, 7:5); UV (DMSO) $\lambda_{\max }(\log \varepsilon) 211(4.84) \mathrm{nm}$; IR $\left(\mathrm{u} \mathrm{cm}^{-1}\right)$ : 2924, 1670, 1591, 1443, 1245, 640; ${ }^{1} \mathrm{H}-\mathrm{NMR}$ (d ppm, DMSO-d ): 1.5 (d, 3H, J=7 Hz, CH-CH $\mathrm{CH}_{3}$ ) 3.4 (s, 3H, N-CH ${ }_{3}$ ) 3.7 (q, 1H, H-2), 7.7 (d, 1H, $\mathrm{J}=8.5 \mathrm{~Hz}, \mathrm{H}-8$ ), 7.9 (dd, $1 \mathrm{H}, \mathrm{J}=8.5$ and $2.2 \mathrm{~Hz}, \mathrm{H}-7$ ), 8.0 (d, $1 \mathrm{H}, \mathrm{J}=2.2 \mathrm{~Hz}$, $\mathrm{H}-5) ;{ }^{13} \mathrm{C}-\mathrm{NMR}(\mathrm{d}$ ppm, DMSO-d $): 19.9\left(\mathrm{CH}_{3}, \mathrm{CH}-\mathrm{CH}_{3}\right), 32.1\left(\mathrm{CH}_{3}, \mathrm{~N}-\mathrm{CH}_{3}\right)$, 40.5 (CH, C-2), 116.4 (CH, C-5), 117.5 (CH, C-7), 128.5 (CH, C-8), 131.9 (C, C-9), 140.4 (C, C-10), 146.6 (C, C-6), 165.1 (C, C-3); ESMS m/z (\%): 238 (24), 195 (100), 166 (13), 154 (54), 95 (12). Analysis calculated for $\mathrm{C}_{9} \mathrm{H}_{8} \mathrm{~N}_{2} \mathrm{O}_{3} \mathrm{~S}: \mathrm{C}, 50.41 ; \mathrm{H}, 4.23 ; \mathrm{N}, 11.76 ; \mathrm{S}, 13.46$. Found: $\mathrm{C}, 50.37 ; \mathrm{H}, 4.27$; $\mathrm{N}, 11.73 ; \mathrm{S}, 14.00$.

\section{General method for the synthesis of compounds 5a-f}

2-substituted-4-methyl-6-nitro-benzo[b][1,4] thiazin-3(4H)-one (4a-b) $(0.01 \mathrm{~mol})$ and hydrazine derivative $(0.01 \mathrm{~mol})$ were dissolved in $15 \mathrm{ml}$ methanol. About $10 \mathrm{ml}$ concentration, $\mathrm{HCl}$ was added into reaction mixture and heated on a steam bath at $70-80^{\circ} \mathrm{C}$ for $2 \mathrm{hrs}$. The reaction mixture was concentrated and cooled in an ice bath.

\section{1-(4-Methyl-6-nitro-2H-benzo $\quad[\mathrm{b}][1,4]$ thiazin-3(4H)-ylidene)} hydrazine (5a)

The title compound was prepared from 4-methyl-6-nitro- $2 \mathrm{H}$ benzo[b][1,4]thiazin-3(4H)-one (4a) and hydrazine hydrate. Product was extracted with cyclohexane $(3 \times 50 \mathrm{ml})$ and purified by column chromatography on silica gel with a mixture of toluene-ethyl acetate (8:2) as eluent. Light yellow oil; yield, 52; $\mathrm{R}_{f} 0.52$ (benzene-acetone, 1:3); UV (DMSO) $\lambda_{\max }(\log \varepsilon) 217$ (4.77) nm; IR $\left(\mathrm{v} \mathrm{cm}^{-1}\right): 3315,2899$, 1688, 1590, 646; ${ }^{1} \mathrm{H}-\mathrm{N}-\mathrm{NMR}$ (d ppm, DMSO-d, $400 \mathrm{MHz}$ ): 2.77 (s, 3H, $\left.\mathrm{N}-\mathrm{CH}_{3}\right), 2.83(\mathrm{~s}, 2 \mathrm{H}, \mathrm{H}-2), 7.36(1 \mathrm{H}, \mathrm{d}, \mathrm{J}=8.6 \mathrm{~Hz}, \mathrm{H}-8), 7.59(1 \mathrm{H}, \mathrm{d}, \mathrm{J}=2.5 \mathrm{~Hz}$, $\mathrm{H}-5), 7.91(1 \mathrm{H}, \mathrm{dd}, \mathrm{J}=2.5,8.6 \mathrm{~Hz}, \mathrm{H}-7), 8.23\left(\mathrm{~s}, 2 \mathrm{H}, \mathrm{NH}_{2}\right) ;{ }^{13} \mathrm{C}-\mathrm{NMR}(\mathrm{d}$ ppm, DMSO-d, $100 \mathrm{MHz}): 30.0\left(\mathrm{CH}_{3}, \mathrm{~N}-\mathrm{CH}_{3}\right), 31.1\left(\mathrm{CH}_{2}, \mathrm{C}-2\right), 107.6$ (CH, C-5), 109.8 (CH, C-7), 123.3 (C, C-9), 128.6 (CH, C-8), 140.7 (C, C-10), 145.6 (C, C-6), 153.7 (C, C-3); ESMS m/z (\%): 238 (11), 199 (39), 164 (24), 138 (100), 102 (16), 88 (11), 74(5). Analysis calculated for $\mathrm{C}_{9} \mathrm{H}_{11} \mathrm{~N}_{3} \mathrm{O}_{2} \mathrm{~S}: \mathrm{C}, 45.37 ; \mathrm{H}, 4.23 ; \mathrm{N}, 23.51 ; \mathrm{S}, 13.46$. Found: $\mathrm{C}, 45.33 ; \mathrm{H}, 4.26$; $\mathrm{N}, 23.55 ; \mathrm{S}, 13.44$.

\section{1-(4-methyl-6-nitro-2H-benzo[b][1,4]thiazin-3(4H)-ylidene)-2- phenylhydrazine (5b)}

The title compound was prepared from 4-methyl-6-nitro- $2 H$-benzo[b] $[1,4]$ thiazin-3 $(4 H)$-one (4a) and phenyl hydrazine hydrochloride and recrystallized from ethanol. Brown crystals; yield, $66 \%$; m.p. $190-192^{\circ} \mathrm{C}$; $\mathrm{R}_{\mathrm{f}} 0.50$ (benzene-acetone, 1:3); UV (DMSO) $\lambda_{\max }(\log \varepsilon) 211$ (4.84) nm; IR ( $\left.\mathrm{v} \mathrm{cm}^{-1}\right)$ : 3362, 2898, 1685, 1592, 1442, 1245,$639 ;{ }^{1} \mathrm{H}-\mathrm{NMR}$ (d ppm, DMSO-d $\left.{ }_{6}, 400 \mathrm{MHz}\right): 2.73$ (s, 3H, N-CH $\left.{ }_{3}\right), 2.91(\mathrm{~s}, 2 \mathrm{H}, \mathrm{H}-2), 6.45(2 \mathrm{H}, \mathrm{dd}$, $\left.J=1.6, J=8.5 \mathrm{~Hz}, \mathrm{H}-2^{\prime}, 6^{\prime}\right), 6.65-7.19\left(3 \mathrm{H}, \mathrm{m}, \mathrm{H}-3^{\prime}, \mathrm{H}-4^{\prime}, \mathrm{H}-5^{\prime}\right), 7.22(1 \mathrm{H}, \mathrm{d}$, $J=8.6 \mathrm{~Hz}, \mathrm{H}-8), 7.31(1 \mathrm{H}, \mathrm{d}, \mathrm{J}=2.5 \mathrm{~Hz}, \mathrm{H}-5), 7.41(1 \mathrm{H}, \mathrm{dd}, \mathrm{J}=2.5,8.6 \mathrm{~Hz}$, H-7), 10.45 (s, 1H, NH); ${ }^{13} \mathrm{C}-\mathrm{NMR}$ (d ppm, DMSO-d ${ }_{6}, 100 \mathrm{MHz}$ ): 30.1 $\left(\mathrm{CH}_{3}, \mathrm{~N}-\mathrm{CH}_{3}\right), 31.5\left(\mathrm{CH}_{2}, \mathrm{C}-2\right), 109.5(\mathrm{CH}, \mathrm{C}-5), 116.3(\mathrm{CH}, \mathrm{C}-2$ ', C-6'), 118.8 (C, C-4'), 119.7 (CH, C-7), 123.2 (C, C-9), 127.8 (CH, C-8), 129.7 (CH, C-3', C-5'), 140.2 (C, C-10), 147.1 (C, C-1'), 153.3 (C, C-3, C-6); ESMS m/z (\%): 314 (14), 275 (7), 226 (11), 200 (39), 164 (24), 150 (100), 136 (12). Analysis calculated for $\mathrm{C}_{15} \mathrm{H}_{13} \mathrm{~N}_{5} \mathrm{O}_{6} \mathrm{~S}: \mathrm{C}, 57.31 ; \mathrm{H}, 4.49 ; \mathrm{N}, 17.82$; S, 10.20. Found: C, 57.35; H, 4.45; N, 17.86; S, 10.18.
1-(4-methyl-6-nitro-2H-benzo[b][1,4]thiazin-3(4H)-ylidene)-2(2,4-dinitrophenyl) hydrazine (5c)

The title compound was prepared from 4-methyl-6-nitro- $2 H$-benzo[b] $[1,4]$ thiazin-3 $(4 H)$-one (4a) and 2,4-m-dinitrophenyl hydrazine and recrystallized from ethanol. Orange crystals; yield, 78\%; m.p. 140$142^{\circ} \mathrm{C}$; $\mathrm{R}_{\mathrm{\rho}}$ value: 0.54 (benzene-acetone, 1:3); UV (DMSO) $\lambda_{\max }(\log \varepsilon)$ 211 (4.84) nm; IR $\left(v^{-1}\right): 3310,2923,1645,1577,1404,1249,1093$, 656; ${ }^{1} \mathrm{H}-\mathrm{NMR}$ (d ppm, DMSO-d, $400 \mathrm{MHz}$ ): 2.72 (s, 3H, N-CH $\left.{ }_{3}\right), 2.81$ (s, $2 \mathrm{H}, \mathrm{H}-2), 7.22(1 \mathrm{H}, \mathrm{d}, \mathrm{J}=8.6 \mathrm{~Hz}, \mathrm{H}-8), 7.44(1 \mathrm{H}, \mathrm{dd}, \mathrm{J}=2.5,8.6 \mathrm{~Hz}, \mathrm{H}-7)$, $7.48(1 \mathrm{H}, \mathrm{d}, \mathrm{J}=2.5 \mathrm{~Hz}, \mathrm{H}-5), 8.03\left(1 \mathrm{H}, \mathrm{d}, \mathrm{J}=8.7 \mathrm{~Hz}, \mathrm{H}-6^{\prime}\right), 8.68(1 \mathrm{H}, \mathrm{dd}$, J=2.6, $8.8 \mathrm{~Hz}, \mathrm{H}-5^{\prime}$ ), 8.89 (1H, d, J=2.6 Hz, H-3'), 11.34 (br, 1H, NH); ${ }^{13} \mathrm{C}$-NMR(d ppm, DMSO-d, $\left.100 \mathrm{MHz}\right): 30.2\left(\mathrm{CH}_{3}, \mathrm{~N}-\mathrm{CH}_{3}\right), 31.3\left(\mathrm{CH}_{2}\right.$, C-2), 109.4 (CH, C-5), 119.4 (CH, C-6', C-7), 121.1 (CH, C-3'), 123.3 (C, C-9, C-2'), 127.0 (CH, C-5'), 127.7 (CH, C-8), 140.3 (C, C-10), 142.7 (C, C-4'), 147.4 (C, C-1'), 153.6 (C, C-3, C-6); ESMS m/z (\%): 404 (10), 365 (38), 316 (44), 290 (25), 254 (100), 136 (8). Analysis calculated for $\mathrm{C}_{15} \mathrm{H}_{13} \mathrm{~N}_{5} \mathrm{O}_{6} \mathrm{~S}$ : C, 44.55; H, 2.99; N, 20.78; S, 7.93. Found: C, 44.57; H, 2.96; N, 20.75; S, 7.96.

\section{1-(2,4-Dimethyl-6-nitro-2H-benzo[b][1,4]thiazin-3(4H)-ylidene) hydrazine (5d)}

The title compound was prepared from 2,4-dimethyl-6-nitro- $2 \mathrm{H}$ benzo[b][1,4]thiazin-3(4H)-one (4b) and hydrazine hydrate. Product was extracted with cyclohexane $(3 \times 50 \mathrm{ml})$ and purified by column chromatography on silica gel with a mixture of toluene-ethyl acetate (8:2) as eluent. Light yellow oil; yield, $70 \% ; \mathrm{R}_{p} 0.42$ (toluene-ethylacetate 3:2); UV (DMSO) $\lambda_{\max }(\log \varepsilon) 225$ (4.49) nm; IR $\left(\mathrm{v} \mathrm{cm}^{-1}\right): 3362,2828$, 1692, 1523, 1245, 659; ${ }^{1} \mathrm{H}-\mathrm{NMR}$ (d ppm, DMSO-d, $400 \mathrm{MHz}$ ): 1.19 (d, $\left.3 \mathrm{H}, \mathrm{J}=7 \mathrm{~Hz}, \mathrm{CHCH}_{3}\right), 2.42\left(\mathrm{~s}, 3 \mathrm{H}, \mathrm{N}-\mathrm{CH}_{3}\right), 2.53$ (q, 1H, H-2), $7.21(1 \mathrm{H}, \mathrm{d}$, $J=8.6 \mathrm{~Hz}, \mathrm{H}-8), 7.28(1 \mathrm{H}, \mathrm{d}, \mathrm{J}=2.5 \mathrm{~Hz}, \mathrm{H}-5), 7.41(1 \mathrm{H}, \mathrm{dd}, \mathrm{J}=2.5,8.6 \mathrm{~Hz}$, $\mathrm{H}-7), 8.51\left(\mathrm{~s}, 2 \mathrm{H}, \mathrm{NH}_{2}\right.$ ); ${ }^{13} \mathrm{C}-\mathrm{NMR}$ (d ppm, DMSO-d $\mathrm{d}_{6} 100 \mathrm{MHz}$ ): 15.0 $\left(\mathrm{CH}_{3}, \mathrm{CHCH}_{3}\right), 30.1\left(\mathrm{CH}_{3}, \mathrm{~N}-\mathrm{CH}_{3}\right), 34.6(\mathrm{CH}, \mathrm{C}-2), 107.7$ (CH, C-5), 109.6 (CH, C-7), 123.3 (C, C-9), 128.0 (CH, C-8), 139.3 (C, C-10), 149.5 (C, C-6), 153.6 (C, C-3); ESMS m/z (\%): 252 (16), 213 (39), 164 (100), 138 (11), 102 (55), 88 (4), 74(5). Analysis calculated for $\mathrm{C}_{9} \mathrm{H}_{11} \mathrm{~N}_{3} \mathrm{O}_{2} \mathrm{~S}: \mathrm{C}, 47.61 ; \mathrm{H}$, 4.79; N, 22.21; S, 12.71. Found: C, 47.58; H, 4.76; N, 22.24; S, 12.74 .

1-(2,4-dimethyl-6-nitro-2H-benzo[b][1,4]thiazin-3(4H)-ylidene)2-phenylhydrazine (5e)

The title compound was prepared from 2,4-dimethyl-6-nitro$2 H$-benzo[b][1,4]thiazin-3(4H)-one $(4 \mathrm{~b})$ and phenyl hydrazine hydrochloride and recrystallized from ethanol. Brown crystals; yield, $68 \%$; m.p. $95-97^{\circ} \mathrm{C} ; \mathrm{R}_{p}, 0.45$ (toluene-ethylacetate 3:2); UV (DMSO) $\lambda_{\max }(\log \varepsilon) 218(4.25) \mathrm{nm} ; \mathrm{IR}\left(\mathrm{v} \mathrm{cm}{ }^{-1}\right): 3393,2899,1701,1593,1525$, 1443, 1245, 828, 637; ${ }^{1} \mathrm{H}-\mathrm{NMR}$ (d ppm, DMSO-d $400 \mathrm{MHz}$ ): 1.20 (d, 3H, J=7 Hz, $\left.\mathrm{CHCH}_{3}\right), 2.54$ (s, 3H, N-CH $)_{3}, 2.84(\mathrm{q}, 1 \mathrm{H}, \mathrm{H}-2), 6.47(2 \mathrm{H}$, $\left.\mathrm{dd}, \mathrm{J}=1.6, \mathrm{~J}=8.5 \mathrm{~Hz}, \mathrm{H}-2^{\prime}, 6^{\prime}\right), 6.58-7.07\left(3 \mathrm{H}, \mathrm{m}, \mathrm{H}-3^{\prime}, \mathrm{H}-4^{\prime}, \mathrm{H}-5^{\prime}\right), 7.22(1 \mathrm{H}$, d, J=8.6 Hz, H-8), $7.31(1 \mathrm{H}, \mathrm{d}, \mathrm{J}=2.5 \mathrm{~Hz}, \mathrm{H}-5), 7.41(1 \mathrm{H}, \mathrm{dd}, \mathrm{J}=2.5,8.6 \mathrm{~Hz}$, $\mathrm{H}-7$ ), 10.50 (s, $1 \mathrm{H}, \mathrm{NH}$ ); ${ }^{13} \mathrm{C}-\mathrm{NMR}$ (d ppm, DMSO-d, $100 \mathrm{MHz}$ ): 15.3 $\left(\mathrm{CH}_{3}, \mathrm{CHCH}_{3}\right), 30.2\left(\mathrm{CH}_{3}, \mathrm{~N}-\mathrm{CH}_{3}\right), 35.9(\mathrm{CH}, \mathrm{C}-2), 107.5(\mathrm{CH}, \mathrm{C}-5), 109.6$ (CH, C-7), 116.2 (CH, C-2', C-6'), 118.8 (C, C-4'), 123.2 (C, C-9), 127.8 (CH, C-8), 129.6 (CH, C-3', C-5'), 140.0 (C, C-10), 147.1 (C, C-1'), 150.4 (C, C-6), 153.4 (C, C-3); ESMS m/z (\%): 328 (8), 289 (14), 240 (40), 214 (7), 178 (24), 150 (100), 136 (12). Analysis calculated for $\mathrm{C}_{15} \mathrm{H}_{13} \mathrm{~N}_{5} \mathrm{O}$ S: C, 58.52; H, 4.91; N, 17.06; S, 9.76. Found: C, 58.57; H, 4.95; $\mathrm{N}, 17.02 ; \mathrm{S}, 9.72$.

1-(2,4-Dimethyl-6-nitro-2H-benzo[b][1,4]thiazin-3(4H)-ylidene)$2-\left(2^{\prime}, 4^{\prime}\right.$-dinitrophenyl)hydrazine (5f)

The title compound was prepared from 2,4-dimethyl-6-nitro- $2 \mathrm{H}$ benzo[b][1,4]thiazin-3(4H)-one (4b) and 2,4-dinitrophenyl hydrazine and recrystallized from ethanol. Orange crystals; yield, 74\%; m.p. 103$105^{\circ} \mathrm{C} ; \mathrm{R}_{\mathrm{f}} 0.78$ (toluene-ethylacetate $3: 2$ ); UV (DMSO) $\lambda_{\max }(\log \varepsilon$ ) 211 (4.84) nm; IR (v cm$\left.{ }^{-1}\right): 3294,2924,1687,1585,1422,1044,907$; ${ }^{1} \mathrm{H}-\mathrm{NMR}$ (d ppm, DMSO-d $\mathrm{d}_{6}, 400 \mathrm{MHz}$ ): 1.21 (d, 3H, J=7 Hz, $\mathrm{CHCH}_{3}$ ), 2.71 $\left(\mathrm{s}, 3 \mathrm{H}, \mathrm{N}-\mathrm{CH}_{3}\right), 2.87(\mathrm{q}, 1 \mathrm{H}, \mathrm{H}-2), 7.29(1 \mathrm{H}, \mathrm{d}, \mathrm{J}=8.6 \mathrm{~Hz}, \mathrm{H}-8), 7.48(1 \mathrm{H}$, d, J=2.5 Hz, H-5), $7.55(1 \mathrm{H}, \mathrm{dd}, \mathrm{J}=2.5,8.6 \mathrm{~Hz}, \mathrm{H}-7), 8.03(1 \mathrm{H}, \mathrm{d}, \mathrm{J}=8.8 \mathrm{~Hz}$, $\left.\mathrm{H}-6^{\prime}\right), 8.49\left(1 \mathrm{H}, \mathrm{dd}, \mathrm{J}=2.6,8.8 \mathrm{~Hz}, \mathrm{H}-5^{\prime}\right), 8.84\left(1 \mathrm{H}, \mathrm{d}, \mathrm{J}=2.6 \mathrm{~Hz}, \mathrm{H}-3^{\prime}\right), 11.27$ (br, $1 \mathrm{H}, \mathrm{NH}$ ); ${ }^{13} \mathrm{C}-\mathrm{NMR}\left(\mathrm{d}\right.$ ppm, DMSO-d ${ }_{6}, 100 \mathrm{MHz}$ ): $15.3\left(\mathrm{CH}_{3}, \mathrm{CHCH}_{3}\right.$ ), 
$30.4\left(\mathrm{CH}_{3}, \mathrm{~N}-\mathrm{CH}_{3}\right), 35.2(\mathrm{CH}, \mathrm{C}-2), 107.5(\mathrm{CH}, \mathrm{C}-5), 109.6\left(\mathrm{CH}, \mathrm{C}-6^{\prime}, \mathrm{C}-7\right)$, 120.7 (CH, C-3'), 126.7 (CH, C-5'), 127.8 (CH, C-8), 133.0 (C, C-9, C-2'), 140.1 (C, C-10), 142.6 (C, C-4'), 147.4 (C, C-1'), 150.1 (C, C-6), 153.1 (C, C-3); ESMS m/z (\%): 418 (13), 379 (11), 330 (54), 304 (7), 268 (13), 254 (100), 136 (11). Analysis calculated for $\mathrm{C}_{15} \mathrm{H}_{13} \mathrm{~N}_{5} \mathrm{O}$ S: C, 45.93; H, 3.37; N, 20.09; S, 7.66. Found: C, 45.91; H, 3.39; N, 20.05; S, 7.68.

\section{General method for the synthesis of compounds 6a-f}

1-(4-Methyl-6-nitro-2H-benzo[b][1,4]thiazin-3(4H)-ylidene)hydrazine derivative (5a-f) $(0.01 \mathrm{~mol})$ in glacial acetic acid $(20 \mathrm{ml})$ and $30 \%$ hydrogen peroxide $(5 \mathrm{ml}$ ) were added and refluxed for 15 minutes. Heating was stopped and another lot of hydrogen peroxide $(5 \mathrm{ml})$ was added. The reaction mixture was again refluxed for 3-4 hrs. Excess of the solvent was removed by distillation under reduced pressure and poured into crushed ice.

\section{1-(4-Methyl-6-nitro-2 $H$-benzo[b][1,4]thiazin-1,1dioxide-3(4H)-} ylidene)hydrazine (6a)

The title compound was prepared by oxidation of 1-(4-methyl-6nitro-2H-benzo[b][1,4] thiazin-3(4H)-ylidene)hydrazine $\quad(5 \mathrm{a})$ and recrystallized from ethanol. Yellow oil; yield, $36 \%$; m.p. $>300^{\circ} \mathrm{C} ; \mathrm{R}_{\mathrm{p}} 0.83$ (toluene-ethylacetate-ethanol 3:1:3); UV (DMSO) $\lambda_{\text {max }}(\log \varepsilon) 216$ (4.55)

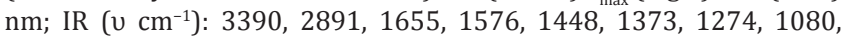
649; ${ }^{1} \mathrm{H}-\mathrm{NMR}$ (d ppm, DMSO-d, $400 \mathrm{MHz}$ ): 2.73 (s, 3H, N-CH ), 3.53 (s, $2 \mathrm{H}, \mathrm{H}-2), 8.11(1 \mathrm{H}, \mathrm{dd}, \mathrm{J}=2.5,8.6 \mathrm{~Hz}, \mathrm{H}-7), 8.36(1 \mathrm{H}, \mathrm{d}, \mathrm{J}=8.6 \mathrm{~Hz}, \mathrm{H}-8)$, 8.59 (1H, d, J=2.5 Hz, H-5), 8.91 (s, $2 \mathrm{H}^{-N_{2}}$ ); ${ }^{13} \mathrm{C}-\mathrm{NMR}$ (d ppm, DMSO-d, $100 \mathrm{MHz}): 30.1\left(\mathrm{CH}_{3}, \mathrm{~N}-\mathrm{CH}_{3}\right), 50.5\left(\mathrm{CH}_{2}, \mathrm{C}-2\right), 109.6(\mathrm{CH}, \mathrm{C}-5), 116.9$ (CH, C-7), 128.2 (CH, C-8), 132.9 (C, C-9), 146.7 (C, C-10), 153.7 (C, C-3, C-6); ESMS m/z (\%): 270 (54), 199 (14), 164 (100), 138 (16), 102 (11), 88 (8), 74(5). Analysis calculated for $\mathrm{C}_{9} \mathrm{H}_{11} \mathrm{~N}_{3} \mathrm{O}_{2} \mathrm{~S}$ : C, 40.00; H, 3.73; N, 20.73; S, 11.86. Found: C, 40.04; H, 3.71; N, 20.71; S, 11.89 .

\section{1-(4-methyl-6-nitro-2 $H$-benzo[b] $[1,4]$ thiazin-1,1 dioxide-3(4H)- ylidene)-2-phenylhydrazine (6b)}

The title compound was prepared by oxidation of 1-(4-methyl-6-nitro$2 H$-benzo[b][1,4]thiazin-3(4H)-ylidene)-2-phenylhydrazine (5b) and recrystallized from ethanol. Brown crystals; yield, $46 \%$; m.p. $>300^{\circ} \mathrm{C}$; $\mathrm{R}_{\mathrm{f}}, 0.37$ (toluene-ethylacetate-ethanol 3:1:3); UV (DMSO) $\lambda_{\max }(\log \varepsilon$ ) 233 (4.39) nm; IR $\left(\mathrm{v} \mathrm{cm}^{-1}\right): 3362,2884,1657,1578,1419,1374,1231$, 1162, 1080, 649; ${ }^{1} \mathrm{H}-\mathrm{NMR}$ (d ppm, DMSO-d ${ }_{6}, 400 \mathrm{MHz}$ ): 2.74 (s, 3H, $\left.\mathrm{N}-\mathrm{CH}_{3}\right), 3.51(\mathrm{~s}, 2 \mathrm{H}, \mathrm{H}-2), 6.46\left(2 \mathrm{H}, \mathrm{dd}, \mathrm{J}=1.6, \mathrm{~J}=8.5 \mathrm{~Hz}, \mathrm{H}-2^{\prime}, 6^{\prime}\right), 6.67-$ $7.22\left(3 \mathrm{H}, \mathrm{m}, \mathrm{H}-3^{\prime}, \mathrm{H}-4^{\prime}, \mathrm{H}-5^{\prime}\right), 8.21(1 \mathrm{H}, \mathrm{dd}, \mathrm{J}=2.5,8.6 \mathrm{~Hz}, \mathrm{H}-7), 8.40(1 \mathrm{H}$, d, J=8.6 Hz, H-8), 8.69 (1H, d, J=2.5 Hz, H-5), $10.64(\mathrm{~s}, 1 \mathrm{H}, \mathrm{NH}) ;{ }^{13} \mathrm{C}-\mathrm{NMR}$ (d ppm, DMSO-d, $100 \mathrm{MHz}$ ): $30.9\left(\mathrm{CH}_{3}, \mathrm{~N}-\mathrm{CH}_{3}\right), 50.9\left(\mathrm{CH}_{2}, \mathrm{C}-2\right), 109.6$

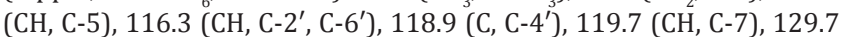
( $\left.\mathrm{CH}, \mathrm{C}-3^{\prime}, \mathrm{C}^{\prime} 5^{\prime}\right), 127.9$ (CH, C-8), 133.2 (C, C-9), 147.2 (C, C-10, C-1'), 153.5 (C, C-3, C-6); ESMS m/z (\%): 346 (12), 275 (6), 226 (52), 200 (38), 164 (23), 150 (100), 136 (12). Analysis calculated for $\mathrm{C}_{15} \mathrm{H}_{13} \mathrm{~N}_{5} \mathrm{O}_{6} \mathrm{~S}$ : C, 52.02; H, 4.07; N, 16.18; S, 9.26. Found: C, 52.04; H, 4.03; N, 16.14; S, 9.29

1-(4-Methyl-6-nitro-2H-benzo[b][1,4]thiazin-1,1 dioxide-3(4H)ylidene)-2-(2',4'-dinitrophenyl)hydrazine (6c)

The title compound was prepared by oxidation of 1-(4-methyl-6nitro-2H-benzo [b][1,4]thiazin-3(4H)-ylidene)-2-(2,4-dinitrophenyl) hydrazine $(5 \mathrm{c})$ and recrystallized from ethanol. Orange crystals; yield, $42 \%$; m.p. $>300^{\circ} \mathrm{C} ; \mathrm{R}_{\mathrm{f}} 0.27$ (toluene-ethylacetate-ethanol 3:1:3); UV (DMSO) $\lambda_{\text {max }}(\log \varepsilon) 235(4.25) \mathrm{nm}$; IR $\left(\mathrm{v} \mathrm{cm}^{-1}\right): 3358,2924,1641,1577$, 1404, 1325, 1248, 1144, 1051, 655; ${ }^{1} \mathrm{H}-\mathrm{NMR}$ (d ppm, DMSO-d ${ }_{6^{\prime}} 400$ MHz): 2.70 (s, 3H, N-CH ${ }_{3}$ ), 3.51 (s, 2H, H-2), $8.05\left(1 \mathrm{H}, \mathrm{d}, \mathrm{J}=8.7 \mathrm{~Hz}, \mathrm{H}-6^{\prime}\right)$, $8.21(1 \mathrm{H}, \mathrm{dd}, \mathrm{J}=2.5,8.6 \mathrm{~Hz}, \mathrm{H}-7), 8.41(1 \mathrm{H}, \mathrm{d}, \mathrm{J}=8.6 \mathrm{~Hz}, \mathrm{H}-8), 8.50(1 \mathrm{H}$, dd, J=2.6, $\left.8.8 \mathrm{~Hz}, \mathrm{H}-5^{\prime}\right), 8.71(1 \mathrm{H}, \mathrm{d}, \mathrm{J}=2.5 \mathrm{~Hz}, \mathrm{H}-5), 8.92(1 \mathrm{H}, \mathrm{d}, \mathrm{J}=2.6 \mathrm{~Hz}$, H-3'), 11.37 (br, 1H, NH); ${ }^{13} \mathrm{C}-\mathrm{NMR}\left(\mathrm{d}\right.$ ppm, DMSO-d ${ }^{\prime}, 100 \mathrm{MHz}$ ): 30.5 $\left(\mathrm{CH}_{3}, \mathrm{~N}-\mathrm{CH}_{3}\right), 50.8\left(\mathrm{CH}_{2}, \mathrm{C}-2\right), 109.6(\mathrm{CH}, \mathrm{C}-5), 119.6\left(\mathrm{CH}, \mathrm{C}-6^{\prime}, \mathrm{C}-7\right)$, 121.2 (CH, C-3'), 127.1 ( $\mathrm{CH}, \mathrm{C}-5^{\prime}$ ), 127.9 (CH, C-8), 133.3 (C, C-9, C-2'), 142.9 (C, C-4'), 147.6 (C, C-10, C-1'), 153.7 (C, C-3, C-6); ESMS m/z (\%): 436 (11), 365 (12), 316 (52), 290 (13), 254 (100), 136 (36). Analysis calculated for $\mathrm{C}_{15} \mathrm{H}_{13} \mathrm{~N}_{5} \mathrm{O}_{6} \mathrm{~S}: \mathrm{C}, 41.29 ; \mathrm{H}, 2.77 ; \mathrm{N}, 19.26 ; \mathrm{S}, 7.35$. Found: $\mathrm{C}$, 41.31; H, 2.73; N, 19.22; S, 7.38.
1-(2,4-Dimethyl-6-nitro-2 $H$-benzo[b] $[1,4]$ thiazin-1,1dioxide$3(4 H)$-ylidene)hydrazine (6d)

The title compound was prepared by oxidation of 1-(2,4-dimethyl-6nitro- $2 H$-benzo[b] $[1,4]$ thiazin-3(4H)-ylidene)hydrazine $(5 \mathrm{~d})$. Product was extracted with cyclohexane $(3 \times 50 \mathrm{ml})$ and purified by column chromatography on silica gel with a mixture of toluene-ethyl acetate (8:2) as eluent. Yellow oil; yield, $31 \% ; R_{r}, 0.28$ (toluene-ethylacetateethanol 3:1:3); UV (DMSO) $\lambda_{\text {max }}(\log \varepsilon) 221$ (4.04) nm; IR $\left(v \mathrm{~cm}^{-1}\right)$ : $3362,2828,1692,1523,1339,1245,1186,1062,660 ;{ }^{1} \mathrm{H}-\mathrm{NMR}$ (d ppm, DMSO-d, $400 \mathrm{MHz}$ ): 1.24 (d, 3H, J=7 Hz, $\left.\mathrm{CHCH}_{3}\right), 2.63\left(\mathrm{~s}, 3 \mathrm{H}, \mathrm{N}-\mathrm{CH}_{3}\right.$ ), 2.93 (q, 1H, H-2), 8.15 (1H, dd, J=2.5, $8.6 \mathrm{~Hz}, \mathrm{H}-7), 8.34$ (1H, d, J=8.6 Hz, $\mathrm{H}-8), 8.60(1 \mathrm{H}, \mathrm{d}, \mathrm{J}=2.5 \mathrm{~Hz}, \mathrm{H}-5), 8.89\left(\mathrm{~s}, 2 \mathrm{H}, \mathrm{NH}_{2}\right) ;{ }^{13} \mathrm{C}-\mathrm{NMR}(\mathrm{d} \mathrm{ppm}$, DMSO-d $\left.{ }_{6}, 100 \mathrm{MHz}\right): 7.3\left(\mathrm{CH}_{3}, \mathrm{CHCH}_{3}\right), 30.7\left(\mathrm{CH}_{3}, \mathrm{~N}-\mathrm{CH}_{3}\right), 49.6(\mathrm{CH}, \mathrm{C}-2)$, 109.7 (CH, C-5), 116.8 (CH, C-7), 128.1 (CH, C-8), 132.9 (C, C-9), 146.6 (C, C-10), 153.6 (C, C-3, C-6); ESMS m/z (\%): 284 (12), 213 (17), 164 (100), 138 (23), 102 (39), 88 (7), 74(5). Analysis calculated for $\mathrm{C}_{9} \mathrm{H}_{11} \mathrm{~N}_{3} \mathrm{O}_{2} \mathrm{~S}: \mathrm{C}, 42.25 ; \mathrm{H}, 4.25 ; \mathrm{N}, 19.71 ; \mathrm{S}, 11.28$. Found: $\mathrm{C}, 42.21 ; \mathrm{H}, 4.30$; N, 19.66; S, 11.22.

1-(2,4-Dimethyl-6-nitro- $2 H$-benzo[b] $[1,4]$ thiazin-1,1dioxide3(4H)-ylidene)-2-phenylhydrazine (6e)

The title compound was prepared by oxidation of 1-(2,4-dimethyl-6nitro-2H-benzo[b][1,4]thiazin-3(4H)-ylidene)-2-phenylhydrazine (5e) and recrystallized from ethanol. Brown crystals; yield, 43\%; m.p. 242$244^{\circ} \mathrm{C} ; \mathrm{R}_{\mathrm{f}} 0.70$ (toluene-ethylacetate-ethanol 3:1:3); UV (DMSO) $\lambda_{\max }$ (log E) $244(4.25) \mathrm{nm}$; IR $\left(\mathrm{v} \mathrm{cm}^{-1}\right): 3393,2938,1670,1586,1449$, 1381.28, 1244, 1162; ${ }^{1} \mathrm{H}-\mathrm{NMR}$ (d ppm, DMSO-d ${ }_{6}, 400 \mathrm{MHz}$ ): 1.23 (d, $\left.3 \mathrm{H}, \mathrm{J}=7 \mathrm{~Hz}, \mathrm{CHCH}_{3}\right), 2.64$ (s, 3H, N-CH $)_{3}, 2.94(\mathrm{q}, 1 \mathrm{H}, \mathrm{H}-2), 6.46(2 \mathrm{H}, \mathrm{dd}$, $\left.\mathrm{J}=1.6, \mathrm{~J}=8.5 \mathrm{~Hz}, \mathrm{H}-2^{\prime}, 6^{\prime}\right), 6.63-7.01\left(3 \mathrm{H}, \mathrm{m}, \mathrm{H}-3^{\prime}, \mathrm{H}-4^{\prime}, \mathrm{H}-5^{\prime}\right), 8.17(1 \mathrm{H}$, dd, J=2.5, 8.6 Hz, H-7), 8.39 (1H, d, J=8.6 Hz, H-8), 8.69 (1H, d, J=2.5 Hz, $\mathrm{H}-5), 10.61$ (s, 1H, NH); ${ }^{13} \mathrm{C}-\mathrm{NMR}$ (d ppm, DMSO-d ${ }_{6}, 100 \mathrm{MHz}$ ): 7.3 $\left(\mathrm{CH}_{3}, \mathrm{CHCH}_{3}\right), 30.8\left(\mathrm{CH}_{3}, \mathrm{~N}-\mathrm{CH}_{3}\right), 49.9(\mathrm{CH}, \mathrm{C}-2), 109.5(\mathrm{CH}, \mathrm{C}-5), 116.2$ (CH, C-2', C-6'), 118.8 (C, C-4'), 119.6 (CH, C-7), 127.8 (CH, C-8), 129.6 (CH, C-3', C-5'), 133.1 (C, C-9), 147.1 (C, C-10, C-1'), 153.4 (C, C-3, C-6); ESMS m/z (\%): 360 (8), 289 (14), 240 (49), 214 (35), 178 (12), 150 (100), 136 (15). Analysis calculated for $\mathrm{C}_{15} \mathrm{H}_{13} \mathrm{~N}_{5} \mathrm{O}_{6} \mathrm{~S}$ : C, 53.32; $\mathrm{H}$, 4.47; N, 15.55; S, 8.90. Found: C, 53.36; H, 4.42; N, 15.59; S, 8.86.

\section{1-(2,4-Dimethyl-6-nitro-2 $H$-benzo[b] [1,4] thiazin-1,1 dioxide-} 3(4H)-ylidene)-2-(2',4'-dinitrophenyl)hydrazine (6f)

The title compound was prepared by oxidation of 1-(2,4-dimethyl-6nitro-2H-benzo[b][1,4] thiazin-3(4H)-ylidene)-2-( $2^{\prime}, 4^{\prime}$-dinitrophenyl) hydrazine (5f) and recrystallized from ethanol. Light brown crystals; yield, 56\%; m.p. $274-276^{\circ} \mathrm{C} ; \mathrm{R}_{\mathrm{p}} 0.78$ (toluene-ethylacetate-ethanol 3:1:3); UV (DMSO) $\lambda_{\text {mx }}(\log \varepsilon) 252(4.20) \mathrm{nm}$; IR $\left(\mathrm{v} \mathrm{cm}^{-1}\right): 3295,2927,1690$, 1590, 1426, 1339, 1248, 1139, 1059, 647; ${ }^{1} \mathrm{H}-\mathrm{NMR}$ (d ppm, DMSO-d $\mathrm{d}^{\prime}$ $400 \mathrm{MHz}): 1.24\left(\mathrm{~d}, 3 \mathrm{H}, \mathrm{CHCH}_{3}\right), 2.65\left(\mathrm{~s}, 3 \mathrm{H}, \mathrm{N}-\mathrm{CH}_{3}\right), 2.95$ (q, 1H, H-2), $8.04\left(1 \mathrm{H}, \mathrm{d}, \mathrm{J}=8.8 \mathrm{~Hz}, \mathrm{H}-6^{\prime}\right), 8.17(1 \mathrm{H}, \mathrm{dd}, \mathrm{J}=2.5,8.6 \mathrm{~Hz}, \mathrm{H}-7), 8.39(1 \mathrm{H}$, d, J=8.6 Hz, H-8), $8.49\left(1 \mathrm{H}, \mathrm{dd}, \mathrm{J}=2.6,8.8 \mathrm{~Hz}, \mathrm{H}-5^{\prime}\right), 8.69(1 \mathrm{H}, \mathrm{d}, \mathrm{J}=2.5 \mathrm{~Hz}$, H-5), $8.89\left(1 \mathrm{H}, \mathrm{d}, \mathrm{J}=2.6 \mathrm{~Hz}, \mathrm{H}-3^{\prime}\right), 11.30(\mathrm{~s}, 1 \mathrm{H}, \mathrm{NH}) ;{ }^{13} \mathrm{C}-\mathrm{NMR}(\mathrm{d}$ ppm, DMSO-d $\left.\mathrm{d}_{6}, 100 \mathrm{MHz}\right): 7.3\left(\mathrm{CH}_{3}, \mathrm{CHCH}_{3}\right), 30.8\left(\mathrm{CH}_{3}, \mathrm{~N}-\mathrm{CH}_{3}\right), 49.9(\mathrm{CH}, \mathrm{C}-2)$, 109.5 (CH, C-5), 119.6 (CH, C-6', C-7), 121.0 (CH, C-3'), 126.9 (CH, C-5'), 127.8 (CH, C-8), 133.1 (C, C-9, C-2'), 142.8 (C, C-4'), 147.5 (C, C-10, C-1'), 153.4 (C, C-3, C-6); ESMS m/z (\%): 450 (13), 379 (12), 330 (52), 304 (8), $268(13), 254(100), 136(10)$. Analysis calculated for $\mathrm{C}_{15} \mathrm{H}_{13} \mathrm{~N}_{5} \mathrm{O} \mathrm{S}$ : C, 42.67; H, 3.31; N, 18.66; S, 7.12. Found: C, 42.61; H, 3.32; N, 18.62; S, 7.16.

\section{Pharmacology}

Test organism and standard drug

All standard drugs (ofloxacin and ketoconazole) were purchased from K K Pharmaceuticals, Udaipur, Rajasthan, whereas all the microorganisms (Escherichia coli, Staphylococcus aureus, Bacillus subtilis, Aspergillus niger, and Candida albicans) were collected from pathology laboratory of RNT Medical College, Udaipur, Rajasthan. All microbes were cultured overnight in nutrient agar medium.

\section{In vitro antimicrobial potential assessment}

In vitro antimicrobial assessment was performed by adopting the disc diffusion method. Representative compounds $5 \mathrm{a}$-f were evaluated for 


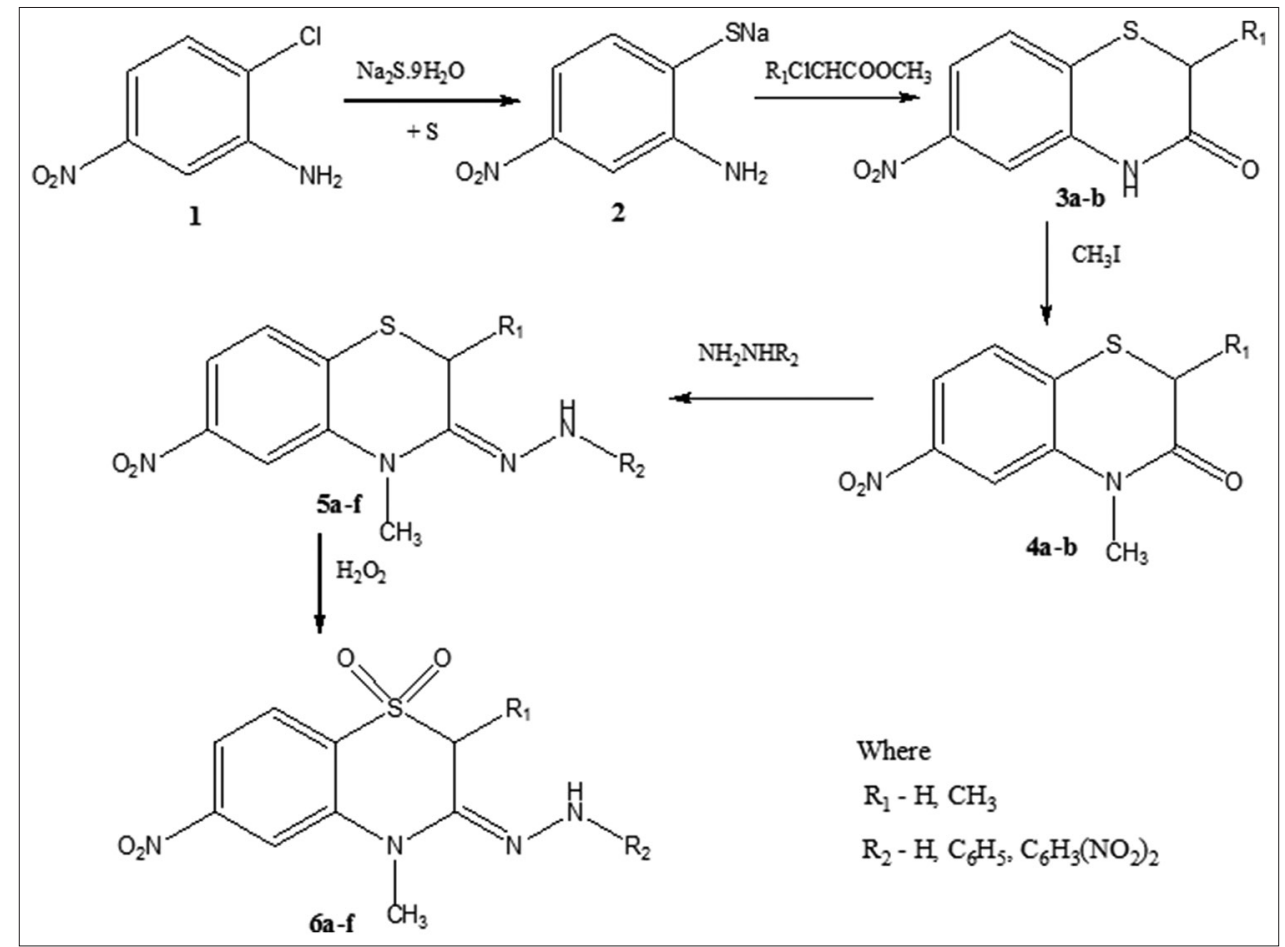

Fig. 1: Synthetic pathway of 1-(4-methyl-6-nitro-2H-benzo[b][1,4]thiazine-3(4H)-ylidene)hydrazine-1,1-dioxide derivatives

their antibacterial against Gram-negative bacteria, E. coli, and Grampositive bacteria, $S$. aureus, B. subtilis, and antifungal activity against A. niger, C. albicans at a concentration of $50 \mu \mathrm{g} / \mathrm{ml}$ using DMSO as a solvent by disc diffusion method. The antibacterial activity was performed with standard drug ofloxacin as positive control and DMSO as negative control after $24 \mathrm{hrs}$ of incubation at $37^{\circ} \mathrm{C}$. The antifungal activity was performed with ketoconazole as positive control and DMSO was used as negative control after $48 \mathrm{hrs}$ of incubation at $25^{\circ} \mathrm{C}$.

\section{Statistical analysis}

The results of the antimicrobial activity of compounds are expressed as mean \pm SD of triplicate samples. Statistically significant differences between groups were measured using one-way analysis of variance followed by two sample t-test of all groups versus their respective control group and ${ }^{*} \mathrm{p}<0.05$ was considered statistically significant, $\mathrm{p}>0.05$ was considered as statistically non-significant, and ${ }^{* *} \mathrm{p}<0.01$ was considered highly significant.

\section{RESULT AND DISCUSSION}

\section{Chemistry}

In IR spectra of all compounds, the bands occur in the region 1404-1379 $\mathrm{cm}^{-1}$ and $1577-1578 \mathrm{~cm}^{-1}$ due to the symmetric and asymmetric stretching vibration of the nitro group. The synthesized 4-methyl-2H-benzo[b][1,4]thiazin-3(4H)-one derivatives 4a-b exhibit a sharp absorption band in the region 2851-2855 due to the $\mathrm{CH}_{3}$ stretching and 1-(4-methyl-6-nitro- $2 H$-benzo[b] [1,4]thiazine-3-yl)hydrazines 5a-f exhibit absorption bands in the region $3360-3100 \mathrm{~cm}^{-1}$ due to the stretching vibration of the secondary amino group. A weak N-N stretching absorption band in the region of $1106-1052 \mathrm{~cm}^{-1}$ and a strong $\mathrm{C}=\mathrm{N}$ stretching absorption band in the $1640-1690 \mathrm{~cm}^{-1}$ region are observed.

${ }^{1}$ HNMR spectra of compounds $5 \mathrm{a}$-f exhibit a multiplet in the region $\delta$ 8.5-6.8 ppm due to aromatic protons. The broad signal observed in the region $\delta 9-11$ is attributed to $-\mathrm{NH}$ protons. The broad peak observed at $\delta$ 2.6-2.8 can be assigned to $-\mathrm{CH}$ proton.

In IR spectra, the synthesized 1-(4-methyl-6-nitro- $2 H$-benzo[b][1,4] thiazine-3(4H)-ylidene)hydrazine-1,1-dioxide derivatives 6a-f exhibit two sharp absorption bands in the region $1195-1135 \mathrm{~cm}^{-1}$ and $1380-$ $1335 \mathrm{~cm}^{-1}$ due to the symmetric and asymmetric stretching vibration of the $\mathrm{SO}_{2}$ group.

In $^{1}$ HNMR spectra, a broad peak observed in the region $\delta$ 8-11 in all 1-(4-methyl-6-nitro-2 $H$-benzo[b][1,4]thiazine-3(4H)-ylidene) hydrazine-1,1-dioxides $6 \mathrm{a}-\mathrm{f}$ is due to $\mathrm{N}-\mathrm{H}$ proton. Aromatic protons show multiplet in the region $\delta 6.8-8.9 \mathrm{ppm}$. The sharp peak observed at $\delta$ 3.2-3.4 can be assigned to $-\mathrm{CH}$ proton. In compounds $6 \mathrm{a}, \mathrm{d}$, a broad peak is observed in the region $\delta 8.2-8.5$ due to $-\mathrm{NH}_{2}$ protons. In compounds $6 \mathrm{~d}, \mathrm{e}, \mathrm{f}$, a doublet peak is observed in the region 1.2-1.3 due to $\mathrm{CH}_{3}$ protons at C-2. ${ }^{13} \mathrm{C}-\mathrm{NMR}$ spectra of compounds $6 \mathrm{a}-\mathrm{f}$ have been recorded. In mass spectra of 1-(4-methyl-6-nitro- $2 H$-benzo[b][1,4] thiazine-3(4H)-ylidene)hydrazine-1,1-dioxides 6a-f, the molecular ion peak is in accordance to their molecular weight.

\section{Biological activity}

Newly synthesized compounds 6a-f exhibited broad-spectrum antimicrobial activity against Gram-positive bacteria, Gram-negative bacterial, and fungal cultures. Antimicrobial activity was measured as the zone of inhibition and represented as mean \pm standard deviation $(n=3)$ in Table 1. Zone of inhibition is depicted in Table 1. After statistical analysis, $p$ value was determined which was significant, that is, $<0.05(\mathrm{p}<0.05)$. It has been noted that compound $6 \mathrm{f}$ having methyl at 2 position and nitro groups at $2^{\prime}$ and $4^{\prime}$ position showed the most potent antibacterial activity, whereas compounds $6 c$ having nitro groups at $2^{\prime}$ and $4^{\prime}$ position and $6 \mathrm{e}$ having methyl at 2 position showed moderate antibacterial activity as compared to reference.

In vitro evaluation of the newly synthesized compounds for the antimicrobial activity is the first step toward achieving the goal of developing a new drug for infectious disease. Earlier, the synthesis of many 1,4-benzothiazine derivatives and their sulfones has been reported to exhibit antimicrobial activity for pharmacological applications. Various hydrazine derivatives [15-17] have been previously reported possessing a broad-spectrum antimicrobial activity. In this research, some new class of sulfones of 1,4-benzothiazines containing different hydrazine derivatives in the 3 -position was screened for antimicrobial properties. The present study through light on the antimicrobial efficacy 
Table 1: Antimicrobial activity of compounds

\begin{tabular}{llllll}
\hline Compound number & \multicolumn{4}{l}{ Antibacterial and antifungal activity at $\mathbf{5 0} \mathbf{\mu g} / \mathbf{m l}$ (zone of inhibition in $\mathbf{m m} \pm \mathbf{S D}$ ) } \\
\cline { 2 - 6 } & E. coli & S. aureus & B. subtilis & C. albicans & A. niger \\
\hline 6a & $14.33 \pm 1.53$ & $16.33 \pm 1.16$ & $15.67 \pm 0.58$ & $12.67 \pm 0.58$ & $13.33 \pm 1.53$ \\
6b & $15.33 \pm 1.16$ & $17.67 \pm 0.58$ & $15.66 \pm 1.53$ & $13.33 \pm 0.58$ & $14.00 \pm 1.73$ \\
6c & $16.66 \pm 1.53$ & $18.67 \pm 0.58$ & $17.33 \pm 1.53$ & $15.00 \pm 0.00$ & $15.33 \pm 0.58$ \\
6d & $15.33 \pm 1.53$ & $16.67 \pm 1.16$ & $15.33 \pm 1.16$ & $13.00 \pm 1.00$ & $14.33 \pm 0.58$ \\
6e & $17.33 \pm 2.52$ & $19.66 \pm 0.58$ & $17.66 \pm 1.53$ & $14.33 \pm 0.58$ & $15.67 \pm 0.58$ \\
6f & $18.33 \pm 2.52$ & $19.66 \pm 1.53$ & $19.33 \pm 1.53$ & $16.67 \pm 0.58$ & $18.33 \pm 1.16$ \\
Control & n. a. & n. a. & n. a. & n. a. & n. a. \\
Ofloxacin & $21.67 \pm 0.58$ & $22.33 \pm 0.58$ & $20.33 \pm 1.53$ & - & - \\
Ketoconazole & - & - & - & $20.33 \pm 0.58$ & $21.67 \pm 2.08$ \\
\hline
\end{tabular}

Values are expressed as mean \pm SD of the three replicates. E. coli: Escherichia coli, S. aureus: Staphylococcus aureus, B. subtilis: Bacillus subtilis, C. albicans: Candida albicans, A. niger: Aspergillus niger, n.a: No activity, SD: Standard deviation

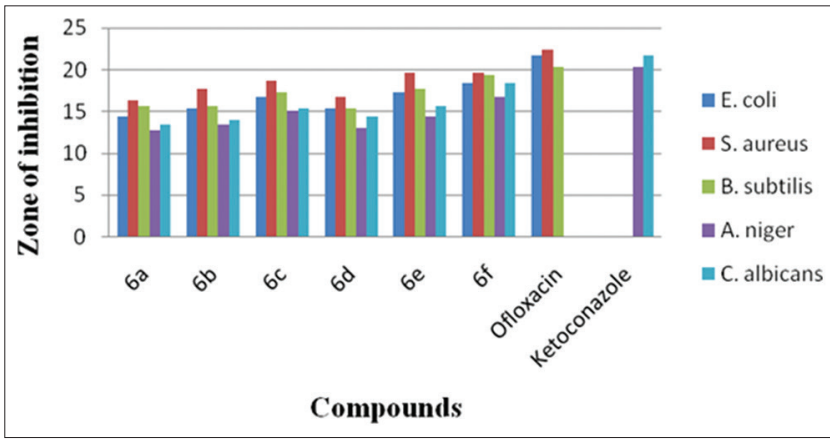

Graph 1: Antimicrobial activity at dose $50 \mu \mathrm{g} / \mathrm{ml}$

of these novel compounds. Result indicated that these synthesized compounds showed more activity toward bacteria as compared to the fungi. Results are collected in Table 1 and Graph 1 [18]

\section{CONCLUSION}

We have reported an easy method to prepare 1-(4-methyl-6-nitro$2 H$-benzo[b][1,4] thiazine-3(4H)-ylidene)hydrazine-1,1-dioxide derivatives, using inexpensive reagents and allowing to introduce different hydrazine derivative in the 3-position. It has been noted that compound of showed the most potent antimicrobial activity, whereas compounds and 6e showed moderate antimicrobial activity as compared to the reference. This study may be helpful for researchers to further development of a new potent antimicrobial drug.

\section{ACKNOWLEDGMENTS}

The authors are thankful to Sophisticated Analytical Instrumentation Facility, Central Instrumentation Laboratory, Punjab University, Chandigarh, for providing spectral and analytical data. The authors are also thankful to the management of B N Institute of Pharmaceutical Sciences, Udaipur, for providing necessary facilities.

\section{REFERENCES}

1. Parai MK, Panda G. A covinient synthesis of chiral amino acid derived 3,4-dihydro-2H-benzo[b][1,4]thiazines and antibiotics levofloxacine. Tetrahedron Lett 2009;50:4703-5.

2. Fringuelli R, Schiaffella F, Utrilla Navarro MP, Milanese L, Santini C, Rapucci M, et al. 1,4-benzothiazine analogues and apoptosis: Structureactivity relationship. Bioorg Med Chem 2003;11(15):3245-54

3. Hori M, Kataoka T, Shmizu H, Imai Y. Ring expansion reactions of benzothizolines to benzo thiazine. Chem Pharm Bull 1973;5:27.

4. Barange DK, Batchu VR, Gorja D, Pattabiraman VR, Tatini LK, Babu JM, et al. Rigioselective construction of six-membered fused heterocyclic rings via $\mathrm{Pd} / \mathrm{C}$-mediated $\mathrm{C}-\mathrm{C}$ coupling followed by iodocyclization strategy: A new entry to $2 \mathrm{H}-1,2-$ benzothiazine-1,1dioxides. Tetrahedron 2007;63:1775-89.

5. Bakavoli M, Nikpour M, Rahimizadeh M, Saberi MR, Sadeghian H. Design and synthesis of pyrimido[4,5-b][1,4]benzothiazine derivatives, as potent 15-lipoxygenase inhibitors. Bioorg Med Chem 2007;15(5):2120-6.

6. Dabholkar VV, Gavande RP. Synthesis of pyrazolyl 1,4-benzothiazine derivatives. Heteroletters 2011;1 Suppl 3:255-61.

7. Saadouni M, Gailane T, Baukhris S, Hassikou A, Habbadi N, Gailane T, et al. Regeoselective synthesis of new variety of 1,4-benzothiazines. Org Commun 2014;7(2):77-84.

8. Montis SD, Fattuoni C, Cadoni E, Cabiddu MG, Usai M, Cabiddu S. High yield synthesis of 4H-1,4-benzothiazine-1,1-dioxide derivatives. J Heterocycl Chem 2008;45:1445-9.

9. Schou SC, Hansen HC, Tangmose TM, Boonen HC, Worsaae A, Drabowski $\mathrm{M}$, et al. Synthesis and pharmacological evaluation of 4H-1,4-benzothiazine-2-carbonitrile-1,1-dioxide and $\mathrm{N}-(2-$ cyanomethyl sulfonylphenyl)acylamide derivatives as potential activators of ATP sensitive potassium channels. Bioorg Med Chem 2005; 13:141-55.

10. Guarda VL, Perrissin M, Thomasson F, Ximenes EA, Galdino SL, Pitta IR, et al. Synthesis and microbial activity of some $2 \mathrm{H}$-benzo[1,4] thiazin-3-one derivatives. Heterocycl Commun 2000;6:49-54.

11. Souza AM, Guarda VL, Leite LF, Filho JM, Lima MC, Galdino SL, et al. On application of knoevenagel condensation for the synthesis of benzo thiazine compounds and structural study. Quim Nov 2006;29:1106-9.

12. Deshmukh MB, Mulik AR, Desal SD. Synthesis of some new 2-methyl1,4-benzothiazine-3(1H)-one derivatives as potential vasodilators. Eur J Chem 2004; 1:206-10.

13. Hamadi MY, Gupta R, Gupta RR. Synthesis and spectral investigations of fluorinated 4H-1,4-benzothiazines and their conversion into sulfones. J Fluorine Chem 1999;94:164-74.

14. Rathore BS, Kumar M. Synthesis of 7-chloro-5-trifluoromrthyl/7fluoro/7-trifluoromethyl-4H-1,4-benzothiazines as antimicrobial agents. Bioorg Med Chem 2006;14:5678-82.

15. Maheshwari M, Goyal A. A review: Synthesis and medicinal importance of 1,4-benzothiazine analogous. Asian J Pharm Clin Res 2015;8:41-6.

16. Karthika C, Mohamed RK, Manivannan S. Phytochemical analysis and evaluation of anti-microbial potential of Senna alata Linn. Leaves extract. Asian J Pharm Clin Res 2016;9:253-7.

17. Maheshwari M, Goyal A. Synthesis and anti-microbial activity of 1-(6-nitro-2H-benzo[b][1,4] thiazine-3(4H)-ylidene)hydrazine-1,1dioxide derivatives. Int J Pharm Pharm Sci 2016;8(10):178-82.

18. Gajbhiye A, Goel KK. Synthesis and anticonvulsant screening of $\mathrm{N}$-aryl-2-(3-oxo-1,4-benzothiazin-2-yl)acetamide derivative. Int $\mathrm{J}$ Pharm Pharm Sci 2013;5(1):220-2. 